\title{
Two-phase flow transient simulation of severe slugging in pipeline-risers systems
}

\author{
G. Hernández, M. Asuaje, F. Kenyery, A. Tremante, O. Aguillón \\ \& A. Vidal \\ Laboratory of Energy Conversion, Simón Bolivar University, Venezuela
}

\begin{abstract}
Pipeline-risers systems are frequently encountered in the petroleum industry, especially in the offshore platforms. Single-phase flow does not involve significant troubles in the operations through these arrangements. However, during multiphase flow, flooding of the separation facilities could be expected due to the generation of severe slugs at the bottom of the riser. The size and frequency of the slugs are functions of the accumulation and displacement of liquid at the base of the riser and can be controlled with an adequate model. An improved transient model is presented to simulate severe slugging phenomenon in pipeline-risers systems. Gas penetration is described thoroughly since the first bubble penetrates into the riser until it reaches the top of it. The model presents improvements in the characteristics method applications including a correction for the gas density deviation caused by the nonfixed space-time resolution during the gas penetration. The results were compared with experimental data and previous models showing better accuracy. The model can be used to design new pipeline riser-systems or to adjust the operation of existing systems to prevent the occurrence of severe slug flow.

Keywords: two-phase flow, pipeline-risers systems, transient model, severe slugging.
\end{abstract}

\section{Introduction}

Severe Slugging process is well known for disturbing operations in pipelinerisers systems due to the production of long liquid slugs in a short period of time, causing flooding of the separation facilities downstream. It occurs when the liquid and gas superficial velocities are relatively low to maintain stratified flow in the pipeline. Once the liquid intends to climb through the riser, the gravity 
force makes difficult the continuity of the process and breaks the steady state condition. Since this moment, accumulation liquid process starts at the bottom of the riser until the gas pressure upstream becomes greater enough. Therefore, this pressure not only overcomes but also penetrates the liquid head, causing the displacement and production of the liquid accumulated in form of severe slugs. The pressure upstream decreases due to gas expansion until it is not enough to hold the remaining liquid in the riser that fall in order to start a new liquid process accumulation. Thus, the production operations remain in an unsteady state or transient conditions.

The severe slugging process has been previously studied. Schmidt et al. [1] described the process in four-step cycle as follows: slug formation, slug movement (production into the separator), blow out and liquid fall back. Taitel et al. [2] presented a model to describe the physical phenomenona but the results were not accurately when compared with experimental data due to the unsatisfied gas continuity in the riser. Fabre et al. [3] developed a model based on the continuous gas penetration through the riser and did not consider the slug formation blocking the gas passage. The model was not able to simulate certain specifics conditions obtained in their own experimental facilities. Sarica and Shoham [4] presented a simplified transient model to describe the phenomenona physically. The simulation of the slug generation, slug production and liquid fall back showed better accuracy than above mentioned models. It is important to remark that the model did not present a procedure to describe the gas penetration into the riser resulting in a cycle time period shorter than experimental data.

This paper presents an improved two-phase transient model to simulate severe slugging phenomenon in pipeline-risers systems where the four-step cycle are described physically. An algorithm is proposed to simulate the gas penetration step, which is considered the most complex. It includes a procedure to correct the gas density deviation caused by the nature of the characteristic method. The model predicts accurately the time period of the cycle along with other variables, which are very important to design separation facilities or to adjust operations in order to ovoid humans and economics risks. The results were compared with Sarica's model [4] showing better performance to simulate the experimental data measured by Fabre et al. [3].

\section{Model description}

In order to simulate the four-step cycle described previously the continuity and momentum equations are formulated for each step of the cycle. The development of the model is based on one-dimensional analysis where the gravity domains and wall shear stress is neglected. This approach can be improved in near future studies when more experimental data become available to simulate highly viscous liquids.

\subsection{Equations during the slug generation step}

The continuity equation for liquid and gas are formulated for this step considering the pipeline void fraction $\alpha_{P}$ as constant. The fig. 1 shows the slug generation process. 


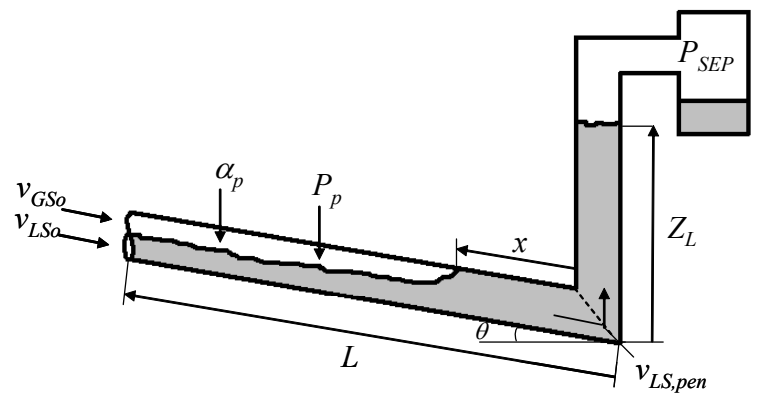

Figure 1: $\quad$ Slug generation process.

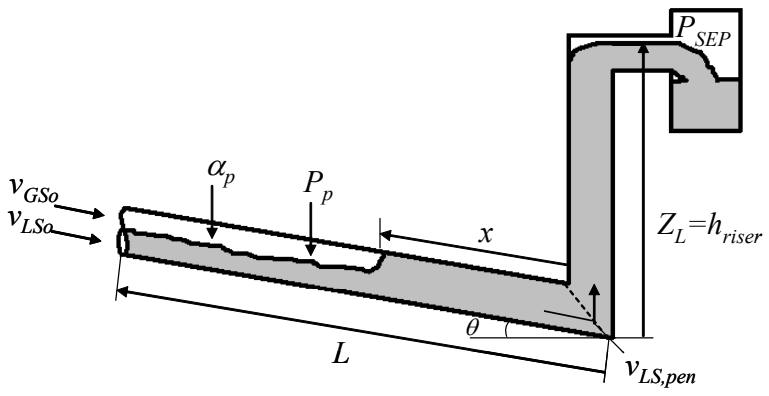

Figure 2: $\quad$ Slug production process.

Taking the pipeline as a volume, control the liquid and gas continuity equations along with the combined momentum equation for this step are respectively:

$$
\begin{gathered}
v_{L S, \text { pen }}=v_{L S, o}-\alpha_{P} \frac{d x}{d t} \\
v_{G S, o} P_{o}=\alpha_{P} \frac{d\left[(L-x) P_{P}\right]}{d t} \\
P_{P}=P_{\text {sep }}+\left(Z_{L}-x \sin \theta\right) \rho_{L} g
\end{gathered}
$$

\subsection{Equations during slug production step}

In this step, the liquid level in the riser $Z_{L}$ has reached the separator and remains constant as shown in the fig. 2.

Thus, eqn. (3) can now be written as follows:

$$
P_{P}=P_{\text {sep }}+\left(h_{\text {riser }}-x \sin \theta\right) \rho_{L} g
$$

Eqns. (2) and (4) can be solved simultaneously, in order to calculate the liquid length and the pressure in the pipeline $x$ and $P_{P}$ respectively. The slug flow rate production into the separator can be calculated with eqn. (1) since the liquid is incompressible. 


$$
q_{L}=\frac{v_{L S, p e n}}{A_{T}}
$$

\subsection{Equations during gas penetration step}

Once the liquid length in the pipe line $x$ is zero from the set of equations presented above, the gas penetration takes place as shown in the fig. $3 \mathrm{a}$.

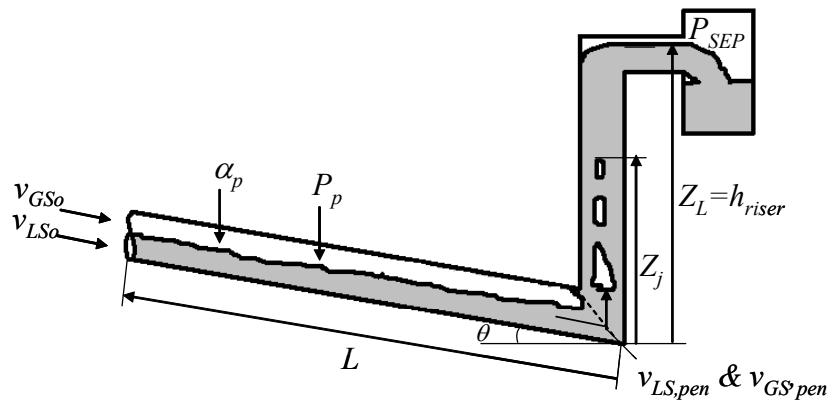

(a)

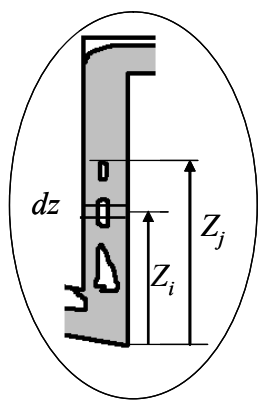

(b)

Figure 3: Gas penetration process.

The boundary conditions at the base of the riser can be obtained from the continuity equations in the pipeline given by

$$
\begin{gathered}
v_{L S, \text { pen }}=v_{L S o} \\
v_{G S, \text { pen }}=\frac{1}{P_{P}}\left(v_{G S, o} P_{o}-\alpha_{P} L \frac{d P_{P}}{d t}\right)
\end{gathered}
$$

Another boundary needed to solve the problem is located at the position of the first bubble that penetrates into the riser $Z_{j}$.

In this step, the system variables $P, \alpha_{r}, \rho_{G}, v_{L S}$ and $v_{G S}$ in the riser are functions of both time and space, while in the pipeline they continue being only function of time. This is due to the no uniformity of the gas void fraction in the riser $\alpha_{r}$ and the hydrostatic pressure.

The continuities for the liquid and gas respectively, and combined momentum equations are formulated for a riser differential control volume shown in the fig. $3 \mathrm{~b}$, and these are:

$$
\begin{gathered}
\frac{\partial\left(1-\alpha_{r}\right)}{\partial t}+\frac{\partial v_{L S}}{\partial z}=0 \\
\frac{\partial\left(\rho_{G} \alpha_{r}\right)}{\partial t}+\frac{\partial\left(\rho_{G} v_{G S}\right)}{\partial z}=0 \\
P_{i}=P_{\text {sep }}+\left(Z_{L}-Z_{j}\right) \rho_{L} g+\left(\int_{Z_{i}}^{Z_{j}} \alpha_{r} d z\right) \rho_{L} g
\end{gathered}
$$


Eqns. (8)-(10) have five unknowns, namely, $P, \alpha_{r}, \rho_{G}, v_{L S}$ and $v_{G S}$ for each control volume position $Z_{i}$. To close the model, two additional equations are needed. Assuming ideal gas behavior one equation would be:

$$
P=\frac{R T \rho_{G}}{M_{G}}
$$

Another equation can be obtained from the drift flux formulation for the flow in the riser given by Zuber and Findlay [5] which is used to obtain the gas void fraction in terms of superficial phase velocities:

$$
\frac{v_{G S}}{\alpha_{r}}=c_{o}\left(v_{G S}+v_{L S}\right)+v_{o}
$$

where $v_{o}=0.35 \sqrt{g d}$ and represents the bubble-rise velocity in stagnant liquid.

\subsection{Equation during gas blowdown}

It occurs since the first gas bubble reaches the top of the riser as shown in the fig. 4. The pipeline pressure decreases drastically in this step until it is not enough to push the thin liquid film remaining in the pipe wall into the separator, causing an instantaneous liquid fall to begin the cycle again. The set of eqns. (8)-(12) can be used to solve the five unknown in this step.

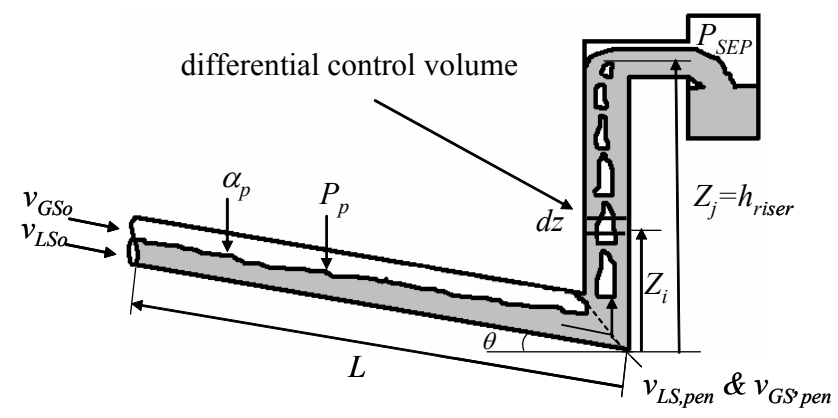

Figure 4: Gas blowdown process.

\section{Characteristic method in the gas penetration step}

In order to calculate $\rho_{G}$ and $\alpha_{r}$ during the gas penetration step, an initial-value problem with a free boundary is formulated and solved with the characteristic method. This method consists to reduce two partial differentials equations into one ordinary differential equation, where characteristic conditions are satisfied. Thus, eqns. (8) and (9) were reduced to the following ordinary differential equation (see appendix A for details),

$$
\frac{D \alpha_{r}}{D t}+\frac{\alpha_{r}}{\rho_{G}}\left(1-\alpha_{r} C_{o}\right) \frac{D \rho_{G}}{D t}=0
$$


Solving this ordinary differential equation, the following constant is obtained

$$
k=\frac{\rho_{G} \alpha_{r}}{\left(C_{o} \alpha_{r}-1\right)}
$$

along with the characteristic:

$$
\frac{d z}{d t}=v_{G}
$$

Eqns. (14) and (15) replace the differentials eqns. (8) and (9) to calculate $\rho_{G}$ and $\alpha_{r}$ as it will be shown in the algorithm furthermore.

\section{Velocities in the gas penetration step}

$v_{L S}$ and $v_{G S}$ are obtained from the same differentials equations (8) and (9), but now through the finite-difference method. Addition of the two mentioned equations gives the following relationship (see appendix A.1 and A.2 for details):

$$
\frac{\partial\left(v_{G S}+v_{L S}\right)}{\partial z}=-\frac{\alpha_{r}}{\rho_{G}}\left(\frac{\partial \rho_{G}}{\partial t}+v_{G} \frac{\partial \rho_{G}}{\partial z}\right)
$$

Substituting eqn. (12) into eqn. (16) the gas superficial velocity is given by

$$
\left(\frac{v_{G S_{z+\Delta z}}{ }^{t+\Delta t}}{\alpha_{r_{z+\Delta z}+\Delta t}{ }^{++\Delta t}}\right)=\frac{\frac{v_{G S_{z}}{ }^{t+\Delta t}}{\alpha_{r z}{ }^{t+\Delta t}}-C_{o} \alpha_{r z+\Delta z}{ }^{t+\Delta t} \frac{\Delta z}{\Delta t}\left[1-\frac{\rho_{G_{z+\Delta z}}{ }^{t+\Delta t}}{\rho_{G_{z+\Delta z}}{ }^{t+\Delta t}}\right]}{1+C_{o} \alpha_{r_{z+\Delta z}{ }^{t+\Delta t}}\left[1-\frac{\rho_{G_{z}}{ }^{t+\Delta t}}{\rho_{G_{z+\Delta z}}{ }^{t+\Delta t}}\right]}
$$

Finally, liquid superficial velocity is obtained from eqn. (12).

\section{Correction of the gas deviation}

For each time-step, the coordinate frame in the riser is different due to the nature of the characteristic method. Analysing eqn. (15), it is obtained $\Delta z=v_{G} \Delta t$, where $\Delta \mathrm{t}$ is established previously as constant and $v_{G}$ increases for each time-step due to the gas expansion. As a result, $\Delta z$ is different for each time-step as shown in the fig. 5 for $t_{5}$ and $t_{6}$.

As a consequence of the nonfixed space-time resolution, a gas density deviation is presented in the eqn. (17) which is formulated under finite-difference method criteria and requires the gas density in the previous time-step, staying in the same position respect to $z$ which does not keep constant. The fig. 6, illustrates better this case.

This happens for all the points during the grid resolution. The correction proposed in this paper is based on the density average between the two closest points e.g. $(3,5)$ and $(4,5)$, around the correct point in the previous step-time, during the grid resolution. This is shown in the algorithm furthermore. 

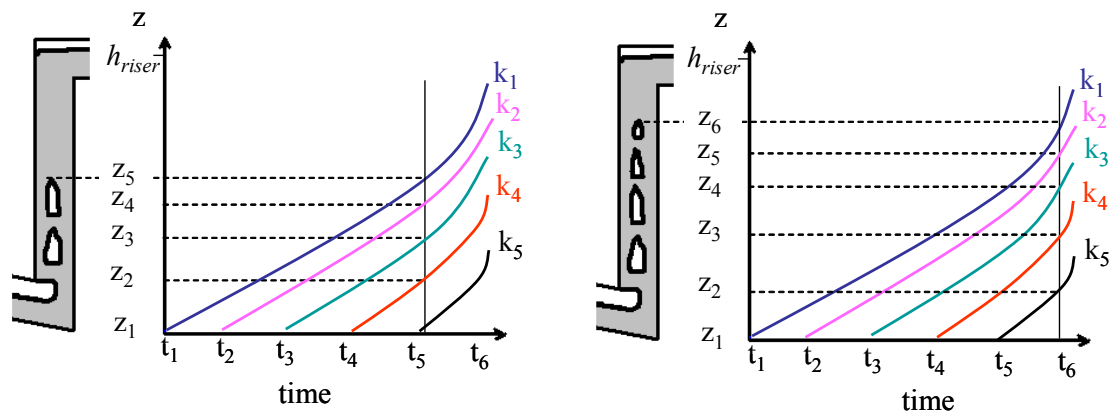

Figure 5: Characteristic method illustrated.

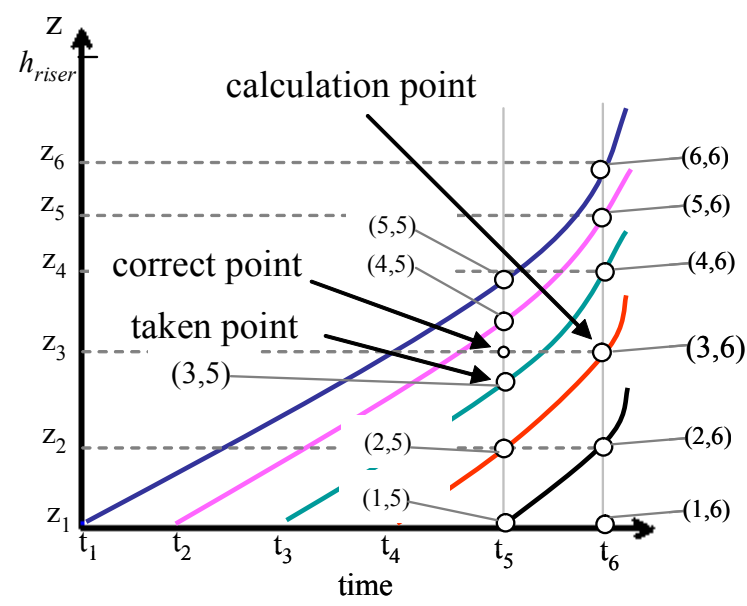

Figure 6: Error presented taking the gas density in the previous time-step, staying in the same position respect to $z$.

\section{Algorithm proposed to simulate gas penetration step}

When the gas is about to penetrate into the riser, the conditions can be determined. Considering $i$ as space counter and $j$ as time counter.

Initial conditions $[i=1$ (the riser base $), j=1(\mathrm{t}=0)]$

1. $v_{L S o}, v_{G S o}$ and $P_{o}$ are given and $\alpha_{P}$ is calculated with any stratified flow model.

2. $z(1,1)=0$, this means the first gas bubble is in the riser base at $\mathrm{t}=0$.

3. $P(1,1)$ and $\rho_{G}(1,1)$ are calculated from eqns. (10) and (11).

4. $\quad v_{L S}(1,1)$ and $v_{G S}(1,1)$ are then calculated from eqns. (6) and (7), $\mathrm{dP}_{\mathrm{p}} / \mathrm{dt}=0$.

5. $\alpha_{r}(1,1)$ is calculated from eqn. (12). 


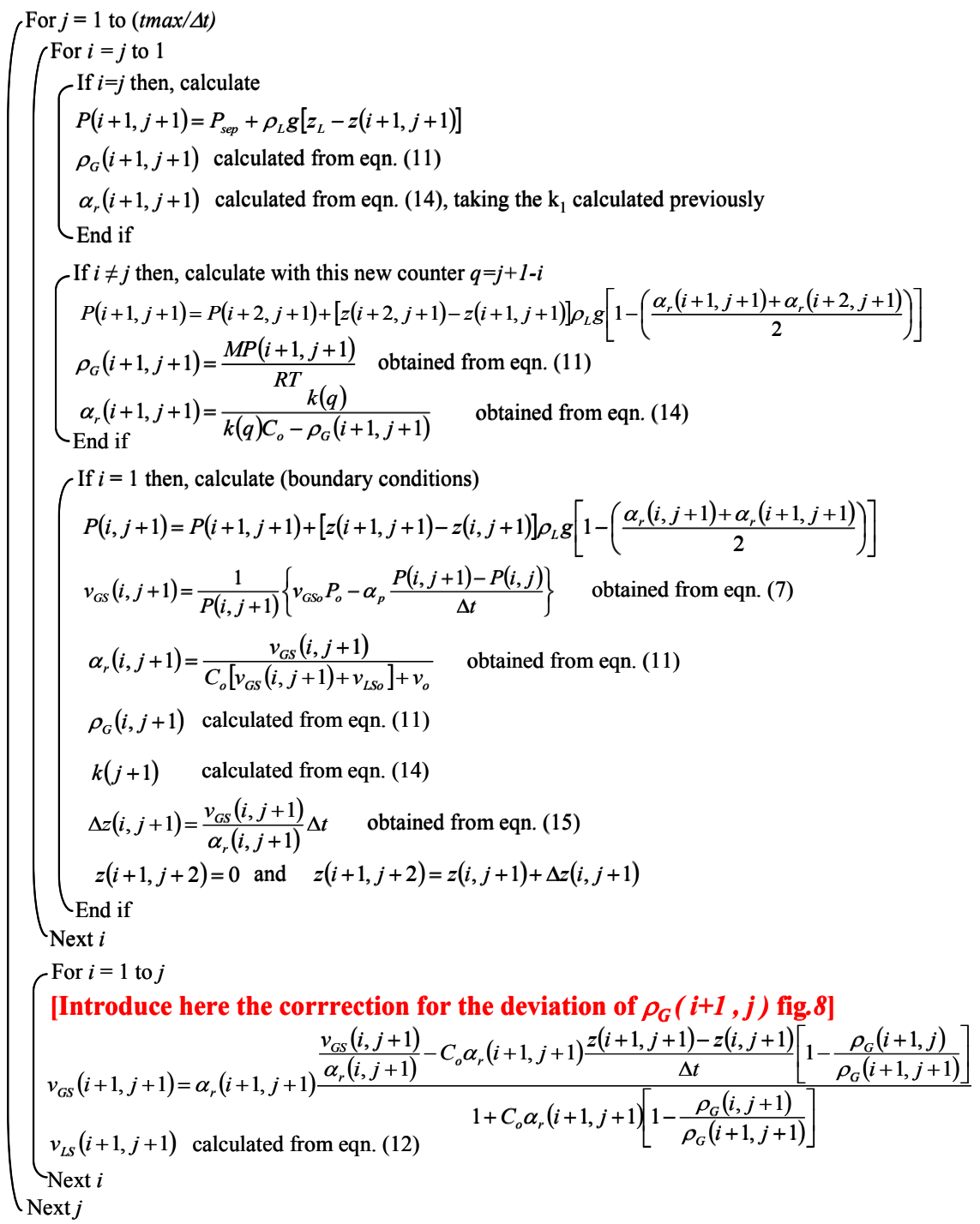

Figure 7: Algorithm proposed to solve the gas penetration step by the characteristic method.

6. $K_{1}$ is obtained from eqn. (14) and will remain constant for the first gas bubble along the time.

7. $\Delta z(1,1)$ is calculated from eqn. (15) with an arbitrary $\Delta t$ established previously.

8. $z(2,2)$ is calculated with $z(1,1)+\Delta z(1,1)$. This is the first gas bubble position in $t+\Delta t$ and represents the second point of the curve described for the first 
bubble on which the solution of the ordinary differential equation $k_{l}$ is satisfied.

9. $\mathrm{z}(1,2)=0$ is established. This means there is another bubble about to penetrate into the riser at $t+\Delta t$.

For the next step time $(t+\Delta t)$ follow the algorithm shown in the fig.7

\section{Comparison of experimental and numerical results}

The numerical results were compared with experimental data of Fabre et al. [3] and Sarica and Shoham [4] model. The data was taking in a laboratory-scale flow loop made of $0.053-\mathrm{m}-\mathrm{ID}$ transparent polyvinyl pipes. The air/water mixture flowed through a $25-\mathrm{m}$-long inclined pipeline with an angle of $-0.57^{\circ}$, and 13.5-m-long vertical riser. The inlet flow conditions were $v_{L S o}=0.13 \mathrm{~m} / \mathrm{s}$ and $v_{G S o}=0.20 \mathrm{~m} / \mathrm{s}$. For the gas, the velocity was calculated from the mass flow rate using the density at $20^{\circ} \mathrm{C}$ and $100 \mathrm{kPa}$. The Sarica and Shoham [4] model was chosen for the comparation because it showed better approximation than the existences models at the moment. The fig. 9 shows the pressure pipeline vs. time in the four-step cycle of severe slugging. Pressure increase corresponds to the slug generation step. Then, the pressure is maintained due to the slug production and finally the pressure decreases due to the gas penetration and gas blow down steps. The same figure compares the results and it can be seen clearly that the two models present good agreement in the slug generation and slug production according to the pipeline pressure. However, there is remarkable difference in the prediction of the gas penetration step due to the rigorous procedure followed through the present model. Finally, the model with the correction of the gas density shows better agreement than the Sarica and Shoham [4] model.

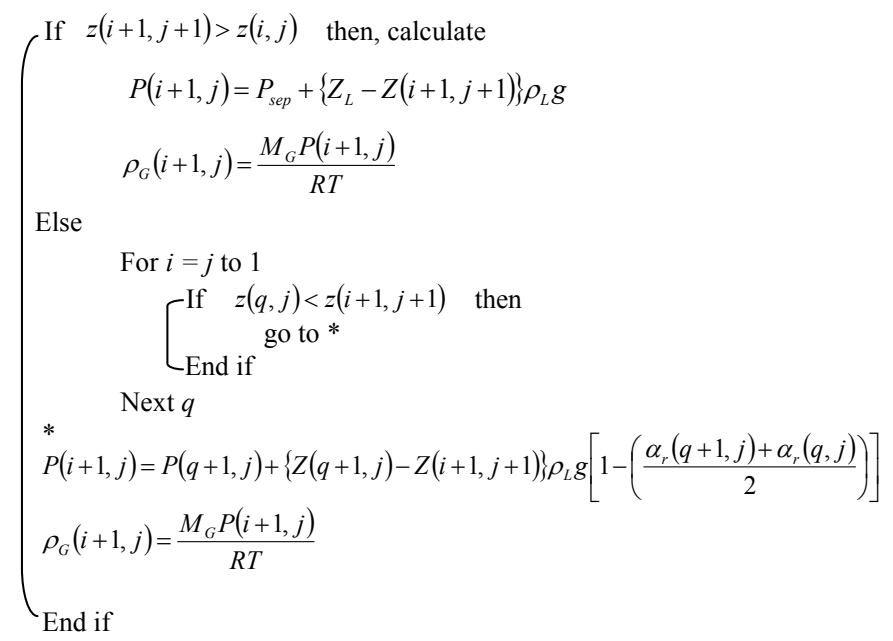

Figure 8: Correction proposed for the gas deviation. 


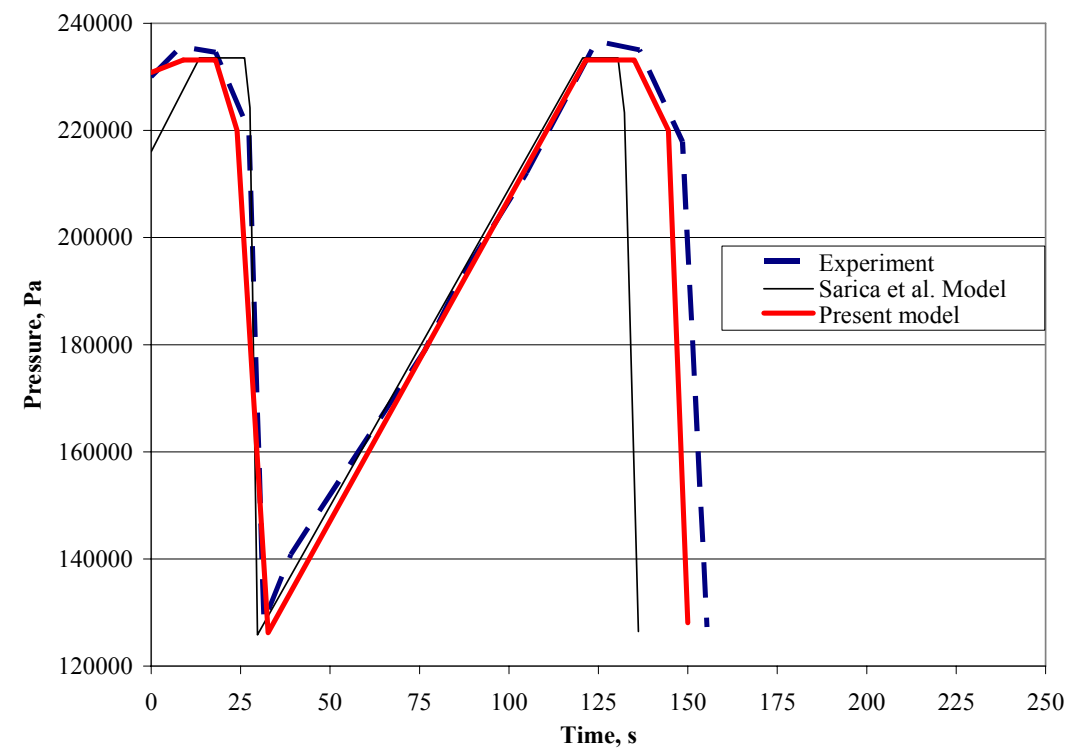

Figure 9: Transient simulation of the pipeline pressure during the severe slugging cycle.

\section{Conclusions}

a. An important contribution has been achieved for transient simulations of two phase flow.

b. A thoroughly algorithm is proposed to simulate transient conditions for the gas penetration step in the severe slugging cycle.

c. Even though the characteristic method is a powerful numerical tool to solve initial-value problems with free-boundary in transient conditions, it requires a correction for the gas deviation generated for the nonfixed space-time resolution.

d. A correction for the characteristic method is proposed in order to simulate the gas penetration step in transient conditions.

e. It has been shown the good agreement of the model when compared with experimental data and Sarica and Shoham [4] model.

f. The four-step severe slugging cycle has been explained physically.

\section{Recommendations}

1. Future studies can adequate the algorithm and the correction proposed for terrain slugging where the riser is not completely vertical.

2. The wall shear stress can be incorporated to the model proposed in order to simulate highly viscous liquids in either severe or terrain slugging cycle. 


\section{Nomenclature}

$v_{L S}$ and $v_{G S}=$ liquid and gas superficial velocities, $\mathrm{m} / \mathrm{s}$

$v_{L S o}$ and $v_{G S o}=$ liquid and gas superficial velocities at the pipeline inlet, $\mathrm{m} / \mathrm{s}$

$\rho_{L}$ and $\rho_{G}=$ liquid and gas densities, $\mathrm{kg} / \mathrm{m}^{3}$

$\alpha_{p}$ and $\alpha_{r}=$ pipeline and riser gas void fraction

$L=$ pipeline length, $\mathrm{m}$

$t=$ time, second

$g=$ acceleration of gravity, $\mathrm{m} / \mathrm{s}^{2}$

$R=$ universal gas constant, $8314.5 \mathrm{Nm} / \mathrm{kmol} \mathrm{K}$

$M_{G}=$ gas molecular mass, $\mathrm{kg} / \mathrm{Kmol}$

$T=$ temperature, $\mathrm{K}$

$d=$ diameter, $\mathrm{m}$

$C_{o}=$ distribution coefficient, 1.2 .

$P_{P}=$ pipeline pressure, $\mathrm{Pa}$

$x=$ coordinate used to measure the liquid length in the pipeline, $\mathrm{m}$

$Z_{L}=$ coordinate used to measure the liquid level in the riser, $\mathrm{m}$

$Z_{j}=$ coordinate used to measure the first gas bubble into the riser, $\mathrm{m}$

$Z_{i}=$ coordinate used to measure the riser differential control volume, $\mathrm{m}$

$P_{i}=$ pressure in a riser point at the coordinate $Z_{i}, \mathrm{~Pa}$

$q_{L}=$ liquid flow rate into the separator, $\mathrm{m}^{3} / \mathrm{s}$

$A_{T}=$ pipe transversal area, $\mathrm{m}^{2}$

\section{Appendix}

Rearranging eqn. (9) gives

$$
\frac{\partial \alpha_{r}}{\partial t}+\frac{\partial v_{G S}}{\partial z}+\frac{\alpha_{r}}{\rho_{G}}\left(\frac{\partial \rho_{G}}{\partial t}+v_{G} \frac{\partial \rho_{G}}{\partial z}\right)=0
$$

Combining eqns. (8) and (A1) yields

$$
\frac{\partial\left(v_{G S}+v_{L S}\right)}{\partial z}=-\frac{\alpha_{r}}{\rho_{G}}\left(\frac{\partial \rho_{G}}{\partial t}+v_{G} \frac{\partial \rho_{G}}{\partial z}\right)
$$

Substituting eqn. (12) in eqn. (A2), the following expression is obtained

$$
\left[\frac{\partial \alpha_{r}}{\partial t}+v_{G} \frac{\partial \alpha_{r}}{\partial z}\right]+\frac{\alpha_{r}}{\rho_{G}}\left(1-\alpha_{r} C_{o}\right)\left[\frac{\partial \rho_{G}}{\partial t}+v_{G} \frac{\partial \rho_{G}}{\partial z}\right]=0
$$

According to the following operator

If

$$
\frac{D()}{D t}=\frac{\partial()}{\partial t}+\frac{d z}{d t} \frac{\partial()}{\partial()}
$$

$$
\frac{d z}{d t}=v_{G}
$$

the terms in the square bracket in eqn. (A3) can be rewritten and eqn. (A3) yields 


$$
\frac{D \alpha_{r}}{D t}+\frac{\alpha_{r}}{\rho_{G}}\left(1-\alpha_{r} C_{o}\right) \frac{D \rho_{G}}{D t}=0
$$

This ordinary differential equation is satisfied along the characteristic direction defined by eqn. (A.5)

\section{References}

[1] Schmidt, Z., Doty, D.R. \& Dutta-Roy, K., Severe slugging in offshore pipeline-riser pipe system. SPE J 12334, pp. 27-38, 1985.

[2] Taitel, Y., Vierkandt, S., Shoham, O. \& Brill, J. P., Severe slugging in a riser system, experimental and modelling. Int. J. Multiphase Flow, 16, pp. 57-68, 1990.

[3] Fabre, J., Peresson, L. L., Corteville, J, Odello, R. \& Bourgeois, T., Severe slugging in pipeline/riser system. SPE 16846, pp. 299-305, 1990

[4] Sarica, C. \& Shoham, O., A simplified transient model for pipeline-riser systems, Chemical Engineering Science. Vol. 46, No. 9, pp. 2167-2179, 1991.

[5] Zuber, N. \& Findlay, J. A., Average volumetric concentration in twophase flow systems. J. Heat Tranfer, Ser. C87, pp. 453-458, 1965. 